\title{
Adjoint of some composition operators on the Dirichlet and Bergman spaces
}

\author{
A. Abdollahi \\ S. Mehrangiz \\ T. Roientan
}

\begin{abstract}
Let $\varphi$ be a holomorphic self-map of the unit disk $\mathbb{U}:=\{z \in \mathbb{C}:|z|<1\}$, and the composition operator with symbol $\varphi$ is defined by $C_{\varphi} f=f \circ \varphi$. In this paper we present formula for the adjoint of composition operators in some Hilbert spaces of analytic functions, in the case that $\varphi$ is a finite Blaschke product or a rational univalent holomorphic self-map of the unit disk $\mathbb{U}$.
\end{abstract}

\section{Introduction}

Let $\varphi$ be a holomorphic self-map of the unit disk $\mathbb{U}:=\{z \in \mathbb{C}:|z|<1\}$. The function $\varphi$ induces the composition operator $C_{\varphi}$, defined on the space of holomorphic functions on $\mathbb{U}$ by $C_{\varphi} f=f \circ \varphi$. The restriction of $C_{\varphi}$ to various Banach spaces of holomorphic functions on $\mathbb{U}$ has been an active subject of research for more than three decades and it will continue to be for decades to come (see [12], [13] and [7]).

The study of composition operator adjoints was initiated more than twenty years ago by Carl Cowen [6], who showed that if $\varphi$ is linear-fractional then $C_{\varphi}^{*}$, the adjoint of $C_{\varphi}$ on $H^{2}$, has the form $M_{g} C_{\sigma} M_{h}^{*}$, where $M_{g}$ and $M_{h}$ are the operators of multiplication by simple rational function $g$ and $h$, bounded on $\mathbb{U}$, and $\sigma$ is a linear-fractional self-map of $\mathbb{U}$ all these functions being easily obtainable from the coefficients of $\varphi$.

Received by the editors in November 2013.

Communicated by H. De Schepper.

2010 Mathematics Subject Classification : Primary 47B33 ; Secondary 47A05.

Key words and phrases : Dirichlet space, composition operator, adjoint, Blaschke product. 
Cowen's work showed, in particular, that one should expect the adjoint of a composition operator to be substantially more complicated than just another composition operator; in the linear-fractional case it is the adjoint of a multiplication operator followed by a weighted composition operator $M_{g} C_{\sigma}$. Complications notwithstanding, Cowen's formula has provided an essential tool in a number of investigations involving linear-fractionally induced composition operators, for example: subnormality and co-subnormality [6], essential normality [2], and self-commutator properties [3].

More recently, Gallardo-Gutiérrez and Montes-Rodríguez in [8] found a nice and simple explicit formula in the Dirichlet space $\mathcal{D}$ for $C_{\varphi}^{*}$, when $\varphi$ is a linear fractional symbol. They have shown that $C_{\varphi}^{*}$ acting on the Dirichlet space is given by the formula

$$
C_{\varphi}^{*} f=f(0) K_{\varphi(0)}-\left(C_{\varphi^{*}} f\right)(0)+C_{\varphi^{*}} f, \quad f \in \mathcal{D} .
$$

In [1], the first author, has considered automorphic composition operators $C_{\varphi}$ acting on the Dirichlet space. By using the E. Gallardo and A. Montes adjoint formula on the Dirichlet space, he has completely determined the spectrum, essential spectrum and point spectrum for self-commutators of such operators.

Martin and Vukotic in [11] have expressed and proved some formulas for the adjoint of $C_{\varphi}$ on the Bergman and Dirichlet spaces, when $\varphi$ is any self-map of $\mathbb{U}$. They have shown that when $\varphi$ is any self-map of $\mathbb{U}, C_{\varphi}^{*}$ acting on the Bergman space is given by the formula

$$
C_{\varphi}^{*} f(w)=\sum_{n=0}^{\infty}\left(\int_{\mathbb{U}} f \bar{\varphi}^{n} d A\right) \cdot(n+1) w^{n}, \quad f \in \mathcal{A}^{2},
$$

and on Dirichlet space is given by the formula

$$
C_{\varphi}^{*} f(w)=f(0) \overline{K_{w}(\varphi(0))}+\int_{T} f(z) \frac{w \overline{z \varphi^{\prime}(z)}}{1-w \overline{\varphi(z)}} d m(z), \quad f \in \mathcal{D} .
$$

In this paper by using these formulas we will express some another formulas on the Dirichlet and Bergman spaces.

\section{Preliminaries}

The Dirichlet space, which we denote by $\mathcal{D}$, is the set of all analytic functions $f$ on the unit disk $\mathbb{U}$ for which

$$
\int_{\mathbb{U}}\left|f^{\prime}(z)\right|^{2} d A(z)<\infty
$$

where $d A$ denote the normalized area measure, equivalently an analytic function $f$ is in $\mathcal{D}$ if $\sum_{n=1}^{\infty} n|\widehat{f}(n)|^{2}<\infty$, where $\widehat{f}(n)$ denotes the $n$th Taylor coefficients of $f$. The inner product inducing the norm of $\mathcal{D}$ is given by

$$
\langle f, g\rangle_{\mathcal{D}}:=f(0) \overline{g(0)}+\int_{\mathbb{U}} f^{\prime}(z) \overline{g^{\prime}(z)} d A(z), \quad f, g \in \mathcal{D} .
$$


The inner product of two functions $f(z)=\sum_{n=0}^{\infty} \widehat{f}(n) z^{n}$ and $g(z)=\sum_{n=0}^{\infty} \widehat{g}(n) z^{n}$ in $\mathcal{D}$ may also be computed by:

$$
\langle f, g\rangle_{\mathcal{D}}=\widehat{f}(0) \overline{\widehat{g}(0)}+\sum_{n=1}^{\infty} n \widehat{f}(n) \overline{\widehat{g}(n)} .
$$

The reproducing kernel consistent with the above product is given by

$$
K_{w}(z)=1+\log \frac{1}{1-\bar{w} z}=1+\sum_{n=1}^{k} \frac{\bar{w}^{n} z^{n}}{n}, w \in \mathbb{U}
$$

In recent years the study of composition operators on the the Dirichlet space has received considerable attention (see [8], [11], [9], [5], [10], and references cited therein).

The weighted Bergman space $A_{\alpha}^{2}$ is the set of all analytic functions in the disk that are square integrable with respect to the weighted area measure $d A_{\alpha}(z)=$ $(\alpha+1)\left(1-|z|^{2}\right)^{\alpha} d A(z),-1<\alpha<\infty$. Here $d A(z)=\pi^{-1} r d r d \theta$, the normalized lebesgue area measure on $\mathbb{U}$. The inner product in this space is given by

$$
\langle f, g\rangle_{A_{\alpha}^{2}}=\int_{\mathbb{U}} f(z) \overline{g(z)} d A_{\alpha}(z)
$$

The standard (unweighted) Bergman space is obtained as a special case: $A^{2}=A_{0}^{2}$. The reproducing kernel in $A_{\alpha}^{2}$ for the point $w$ in the disk is given by

$$
K_{w}(z)=\frac{1}{(1-\bar{w} z)^{2+\alpha}}
$$

with a suitably chosen analytic branch of the power.

Let $\varphi$ be a linear fractional map of $\mathbb{U}$ defined by

$$
\varphi(z)=\frac{a z+b}{c z+d}
$$

such that $a d-b c \neq 0$ and $\varphi(\mathbb{U}) \subset \mathbb{U}$. For such linear fractional map define the associated linear fractional transformation $\varphi^{*}$ by

$$
\varphi^{*}(z)=\frac{\bar{a} z-\bar{c}}{-\bar{b} z+\bar{d}}
$$

For any rational function $R$, expressed as a quotient of relatively prime polynomials, the degree of $R$ is the larger of the degrees of its numerator and denominator. It is an elementary exercise to show that if the degree of $R$ is $d$ then for each point $w \in \mathbb{C}$, the inverse image $R^{-1}(w)$ has, counting multiplicities, exactly $d$ points. If $R^{-1}(w)$ has $d$ distinct points we will say $w$ is a regular value of $R$. 


\section{Adjoints with symbols $\varphi(z)=z \frac{1-z^{n}}{n(1-z)}$ and $\varphi(z)=\frac{z^{n}+a}{1+\bar{a} z^{n}}$}

Let $\varphi(z)=z \frac{1-z^{n}}{n(1-z)}$ or $\varphi(z)=\frac{z^{n}+a}{1+\bar{a} z^{n}},|a|<1$. In this section we are going to find some explicit formulae for the adjoint of $C_{\varphi}$ on the Dirichlet and Bergman spaces.

Theorem 3.1. Let $\varphi(z)=z \frac{1-z^{n}}{n(1-z)}$, for $z \neq 1$ and $\varphi(1)=1$. Then the adjoint formula for $C_{\varphi}$ on the Dirichlet space is given by

$$
C_{\varphi}^{*} f(w)=\sum_{i=1}^{n} f\left(\frac{1}{\overline{z_{i}}}\right)-(n-1) f(0)
$$

where $z_{i}$ are the roots of the equation $1-\bar{w} \varphi(z)=0$.

Proof. Let $f \in \mathcal{D}$. Since

$$
f(w)=\left\langle f, k_{w}\right\rangle_{\mathcal{D}}=\left\langle f, 1+\log \frac{1}{1-\bar{w} \varphi(z)}\right\rangle_{\mathcal{D}}
$$

by formula (1.3), it follows that

$$
\begin{aligned}
C_{\varphi}^{*} f(w) & =f(0)+\int_{T} f(z) \overline{\bar{z}\left(1+\log \frac{1}{1-\bar{w} \varphi(z)}\right)^{\prime}} d m(z) \\
& =f(0)+\int_{T} f(z) \overline{z\left(1+\log \frac{1}{\left(z-z_{1}\right) \ldots\left(z-z_{n}\right)}\right)^{\prime}} d m(z) \\
& =f(0)-\int_{T} f(z) \frac{\bar{z}}{\overline{z-z_{1}}} \frac{d z}{2 \pi i z}-\ldots-\int_{T} f(z) \frac{\bar{z}}{\overline{z-z_{n}}} \frac{d z}{2 \pi i z} \\
& =\sum_{i=1}^{n} f\left(\frac{1}{\bar{z}_{i}}\right)-(n-1) f(0) .
\end{aligned}
$$

Corollary 3.2. Suppose that $\rho: \hat{C} \longrightarrow \hat{C}$ denotes inversion in the unit circle, $\rho(z)=\frac{1}{\bar{z}}$, $\varphi(z)=z \frac{1-z^{n}}{n(1-z)}$, and $w_{0} \in \mathbb{U}$ is a regular value of $\varphi_{e}=\rho \circ \varphi \circ \rho$ and $V \subset \mathbb{U}$ is any connected neighborhood of $w_{0}$ on which are defined $n$ distinct branches $\left\{\sigma_{j}\right\}_{j=1}^{n}$ of $\varphi_{e}^{-1}$. Then for all non zero $w \in V$ we have

$$
C_{\varphi}^{*} f(w)=\sum_{j=1}^{n} f\left(\sigma_{j}(w)\right)-(n-1) f(0) .
$$

and for $w=0$,

$$
C_{\varphi}^{*} f(0)=f(0)
$$

Theorem 3.3. Let $\varphi(z)=\frac{z+z^{2}+\ldots+z^{n}}{n}$. Then the adjoint of $C_{\varphi}$ on the Bergman space is given by

$$
C_{\varphi}^{*} f(w)=\sum_{m=0}^{\infty}\left(\frac{1}{n^{m}} \frac{\left(F(z)\left(1+z+\ldots+z^{n-1}\right)^{m}\right)^{(n m+1)}(0)}{(n m+1) !}\right) \cdot(m+1) w^{m},
$$

where $F(z)$ is holomorphic on the unit disk $\mathbb{U}$, such that for each $z \in \mathbb{U}, F^{\prime}(z)=f(z)$. 
Proof. Let $\varphi(z)=\frac{z+z^{2}+\ldots+z^{n}}{n}$, and $f$ be a polynomial function. Then by using formula (1.2), it follows that

$$
\begin{aligned}
C_{\varphi}^{*} f(w) & =\sum_{m=0}^{\infty}\left(\int_{D} f \bar{\varphi}^{m} d A\right) \cdot(m+1) w^{m} \\
& =\sum_{m=0}^{\infty}\left(\int_{T} F(z)\left(\frac{z^{n-1}+\ldots+1}{n z^{n}}\right)^{m} \frac{d z}{2 \pi i z^{2}}\right) \cdot(m+1) w^{m} \\
& =\sum_{m=0}^{\infty}\left(\frac{1}{n^{m}} \operatorname{Res}\left(\frac{F(z)\left(1+z+\ldots+z^{n-1}\right)^{m}}{z^{m n+2}}, 0\right)\right) \cdot(m+1) w^{m} \\
& =\sum_{m=0}^{\infty}\left(\frac{1}{n^{m}} \frac{\left(F(z)\left(1+z+\ldots+z^{n-1}\right)^{m}\right)^{(n m+1)}(0)}{(n m+1) !}\right) \cdot(m+1) w^{m}
\end{aligned}
$$

The statement for arbitrary $f$ in $\mathcal{A}^{2}$ follows from the density of the polynomials.

Martin and Vukotic in [11] have given the formula for the adjoint of $C_{\varphi}$ on the Hardy space, when $\varphi(z)=z^{n}$. By using the similar proof as the proof of Theorems 3.1 and 3.3, we conclude explicit formulas for the monomial symbols on the Dirichlet and Bergman spaces.

Corollary 3.4. Let $\varphi(z)=z^{n}$ and $C_{\varphi}$ be the composition operator on the Dirichlet space induced by $\varphi$. Then

$$
C_{\varphi}^{*} f(w)=\sum_{k=0}^{n-1} f\left(r_{k, w}\right)-(n-1) f(0),
$$

where $r_{k, w}=r^{\frac{1}{n}} e^{i\left(\frac{\theta+2 k \pi}{n}\right)}, k=0,1, \ldots n-1$ are the $n$th roots of the equation $\varphi(z)=w$.

Corollary 3.5. Let $\varphi(z)=z^{m}$ and $C_{\varphi}$ be the composition operator on the Bergman space induced by $\varphi$. Then

$$
C_{\varphi}^{*} f(w)=\sum_{n=0}^{\infty}(n+1) w^{n} \frac{f^{m n}(0)}{(m n+1) !}
$$

Theorem 3.6. Let $\varphi(z)=\frac{z^{n}+a}{1+\bar{a} z^{n}}$. Then the following formula for the adjoint of $C_{\varphi}$ on the Dirichlet space holds:

If $w \neq 0$,

$$
C_{\varphi}^{*} f(w)=\sum_{k=0}^{n-1}\left(f(0) k_{\psi(0)}+C_{\psi^{*}} f\right)\left(r_{k, w}\right)-n\left(C_{\psi^{*}} f(0)+f(0)\right)+f(0),
$$

and for $w=0$

$$
C_{\varphi}^{*} f(0)=0,
$$

where $\psi(z)=\frac{z+a}{1+\bar{a} z}, \psi^{*}(z)=\frac{z-a}{1-\bar{a} z}$ and $r_{k, w}=r^{\frac{1}{n}} e^{i\left(\frac{\theta+2 k \pi}{n}\right)}, k=0,1, \ldots n-1$ are the $n$th roots of the equation $z^{n}=w$. 
Proof. Let $\phi(z)=z^{n}$ and $\psi(z)=\frac{z+a}{1+\bar{a} z}$. Since $\phi$ is an analytic self-map of $\mathbb{U}$ and $\varphi=\psi \circ \phi$, we have $C_{\varphi}=C_{\phi} \circ C_{\psi}$. Also, the boundedness of $C_{\phi}$ implies the boundedness of $C_{\varphi}$. By a simple computation and using formula (1.1) and Corollary (3.4), for nonzero $w \in \mathbb{U}$ it follows that

$$
C_{\varphi}^{*} f(w)=\sum_{k=0}^{n-1}\left(f(0) k_{\psi(0)}+C_{\psi^{*}} f\right)\left(r_{k, w}\right)-n\left(C_{\psi^{*}} f(0)+f(0)\right)+f(0)
$$

where $r_{k, w}=r^{\frac{1}{n}} e^{i\left(\frac{\theta+2 k \pi}{n}\right)}, k=0,1, \ldots n-1$ are the $n$th roots of the equation $z^{n}=w$, and for $w=0, C_{\varphi}^{*} f(0)=f(0)$.

\section{Adjoint with rational self-map symbols}

Bourdon and Shapiro in [4] express and prove formula for the adjoint of $C_{\varphi}$ on the Hardy space, when $\varphi$ is rational self-map of the unit disk $\mathbb{U}$. In this section for such symbol, we are going to find some formula for the adjoint of $C_{\varphi}$ on the Dirichlet and Bergman spaces.

Theorem 4.1. Let $\varphi$ be any rational self-map of the unit disk $\mathbb{U}$, say of degree $n$. Define the associated rational function

$$
\varphi^{*}(z)=\overline{\varphi\left(\frac{1}{\bar{z}}\right)}, z \in \mathbb{U}
$$

Assume further $\frac{1}{1-\bar{w} \varphi(z)}=\frac{1}{\left(z-\alpha_{i}\right)^{m_{i}} p(z)}$, with $p\left(\alpha_{i}\right) \neq 0$ and put $A_{m_{i}}(z)=c_{1}(z)+$ $c_{2}\left(z^{2}\right)+\ldots+c_{m_{i}}\left(z^{m_{i}}\right)$, where $c_{1}, c_{2}, \ldots c_{m}$ are the Taylor coefficients of $\frac{1}{p(z)}$ in $z=\alpha_{i}$. Then the adjoint formula of $C_{\varphi}$ on the Dirichlet space $\mathcal{D}$ is given by

$$
\begin{aligned}
C_{\varphi}^{*} f(w)=f(0) K_{\varphi(0)}(w) & +\sum_{k} \operatorname{Res}\left(\frac{w f(z) \varphi^{\prime *}(z)}{\left(1-w \overline{\varphi(\infty)} z^{2}\right)}, z_{k}\right) \\
& +\sum_{j=1}^{d} \sum_{k} \operatorname{Res}\left(\frac{w f(z) \varphi^{\prime *}(z) A_{m_{j}}^{\prime}\left(\frac{z}{1-\overline{z_{i}} z}\right)}{z^{2}}, z_{k}\right),
\end{aligned}
$$

where the sum is taken over the zeros $z_{k}$ of the denominator of the $\varphi^{\prime *}(z)$ and $\frac{1}{z^{2}} A_{m_{j}}^{\prime}\left(\frac{z}{1-\overline{z_{i}} z}\right)$, and the zeros of the rational function $1-w \overline{\varphi(\infty)} z^{2}$, which belong to $\mathbb{U}$.

Proof. By using formula (1.3) it follows that

$$
C_{\varphi}^{*} f(w)=f(0) K_{\varphi(0)}(w)+\left\langle f(z), \bar{w} z \frac{\varphi^{\prime}(z)}{1-\bar{w} \varphi(z)}\right\rangle_{H^{2}} .
$$

Let $\left\{z_{1}, z_{2} \ldots z_{d}\right\}$ be the roots of the equation $1-\bar{w} \varphi(z)$ that order of a multiplicity $z_{i}$, is $m_{i}$. By a simple computation we conclude that if $\varphi(z)$ is a rational self-map of the unit disk of degree $n$, then

$$
\frac{1}{1-\bar{w} \varphi(z)}=\frac{1}{1-\bar{w} \varphi(\infty)}+\sum_{j=1}^{d} A_{m_{i}}\left(\frac{1}{z-z_{i}}\right) .
$$


Let $f$ first be a polynomial. So we have

$$
\begin{aligned}
C_{\varphi}^{*} f(w)=f(0) K_{\varphi(0)}(w) & +\left\langle f(z), \bar{w} z \varphi^{\prime}(z)\left(\frac{1}{1-\bar{w} \varphi(\infty)}\right.\right. \\
& \left.\left.+\sum_{j=1}^{d} A_{m_{j}}\left(\frac{1}{z-z_{j}}\right)\right)\right\rangle \\
=f(0) K_{\varphi(0)}(w) & +\int_{T} w f(z) \frac{\overline{z \varphi^{\prime}(z)}}{1-\overline{w \varphi(\infty)}} \frac{d z}{2 \pi i z} \\
& +\sum_{j=1}^{d} \int_{T} w f(z) \overline{z \varphi^{\prime}(z) A_{m_{j}}\left(\frac{1}{z-z_{j}}\right) \frac{d z}{2 \pi i z}} \\
& +\sum_{k} \operatorname{Res}\left(\frac{w f(z) \varphi^{\prime *}(z)}{\left(1-w \overline{\varphi(\infty)} z^{2}\right)}, z_{k}\right) \\
& +\sum_{j=1}^{d} \sum_{k} \operatorname{Res}\left(\frac{w f(z) \varphi^{\prime *}(z) A_{m_{j}}^{\prime}\left(\frac{z}{1-\overline{z_{i} z}}\right)}{z^{2}}, z_{k}\right),
\end{aligned}
$$

where $A_{m_{i}}^{\prime}(z)=\overline{c_{1}}(z)+\overline{c_{2}}\left(z^{2}\right)+\ldots \overline{c_{m_{i}}}\left(z^{m_{i}}\right)$. The statement for arbitrary $f$ in $\mathcal{A}^{2}$ follows from the density of the polynomials.

Corollary 4.2. Let $\varphi$ be any rational self-map of the unite disk $\mathbb{U}$, such that $\varphi(\infty)=\infty$. Then we have

$$
C_{\varphi}^{*} f(w)=\sum_{j=1}^{d} \sum_{k} \operatorname{Res}\left(\frac{w f(z) \varphi^{\prime *}(z) A_{m_{j}}^{\prime}\left(\frac{z}{1-\overline{z_{i}} z}\right)}{z^{2}}, z_{k}\right),
$$

where $A_{m_{j}}$ is as Theorem 4.1 and the sum is taken over the zeros $z_{k}$ of the denominator of the $\varphi^{*}(z)$ and $\frac{1}{z^{2}} A_{m_{j}}^{\prime}\left(\frac{z}{1-\overline{z_{i}} z}\right)$, which belong to $\mathbb{U}$.

Example 4.3. Let $\varphi(z)=z \frac{z-a}{1-\bar{a} z}, f \in \mathcal{D}$ and $0 \neq w \in \mathbb{U}$ be a regular value of $\varphi$. Then the roots $z_{1}$ and $z_{2}$ of equation $\varphi(z)=w$ are distinct. So we have

$$
\frac{1}{\varphi(z)-w}=\frac{1}{g(z)\left(z-z_{1}\right)}
$$

where $g\left(z_{1}\right) \neq 0$. Hence $z_{i}$ is the center of an open disk to which $\frac{1}{g(z)}$ has a holomorphic restriction and so, we have

$$
\frac{1}{\varphi(z)-w}=\frac{1}{g(z)\left(z-z_{1}\right)}=\frac{\beta_{1}}{z-z_{1}}+d_{2}+d_{3}\left(z-z_{1}\right) \ldots
$$

Put

$$
g_{1}(z)=\frac{1}{\varphi(z)-w}-\frac{\beta_{1}}{z-z_{1}}
$$

and by the same work put

$$
g_{2}(z)=\frac{1}{\varphi(z)-w}-\frac{\beta_{1}}{z-z_{1}}-\frac{\beta_{2}}{z-z_{2}} .
$$


Then $g_{2}$ is holomorphic on complex plane. So we have

$$
\frac{1}{\varphi(z)-w}=\frac{\beta_{1}}{z-z_{1}}+\frac{\beta_{2}}{z-z_{2}}
$$

By using formula (1.3), it follows that

$$
\begin{aligned}
C_{\varphi}^{*} f(w)= & f(0) K_{\varphi}(0)(w)+\int_{T} f(z) w \frac{\overline{z \varphi^{\prime}(z)}}{1-w \overline{\varphi(z)}} \frac{d z}{2 \pi i z} \\
= & f(0)+\int_{T} f(z) w \frac{1}{1-\bar{a} z}\left(\frac{\beta_{1}}{z-z_{1}}+\frac{\beta_{2}}{z-z_{2}}\right) \frac{d z}{2 \pi i z} \\
& +\int_{T} f(z) w \frac{1}{z-a}\left(\frac{\beta_{1}}{z-z_{1}}+\frac{\beta_{2}}{z-z_{2}}\right) \frac{d z}{2 \pi i} .
\end{aligned}
$$

We first assume that $f$ is a polynomial. Then

$$
C_{\varphi}^{*} f(w)=f(0)+w \sum_{i=1}^{2}\left(f\left(z_{i}\right) \frac{\beta_{i}}{z_{i}\left(1-\bar{a} z_{i}\right)}-f(0) \frac{\beta_{i}}{z_{i}}+f(a) \frac{\beta_{i}}{a-z_{i}}+f\left(z_{i}\right) \frac{\beta_{i}}{z_{i}-a}\right)
$$

where $\beta_{j}(w)=\frac{1}{\varphi^{\prime}\left(\sigma_{j}(w)\right)}=\sigma_{j}^{\prime}(w)$. Since $w \in \mathbb{U}$ is a regular value of $\varphi$ and $\varphi$ has degree 2 , there is a connected neighborhood $V$ of $w$, on which are defined 2 distinct branches $\left\{\sigma_{j}\right\}_{j=1}^{2}$ of $\varphi^{-1}$. So for non zero $w \in \mathbb{U}$, we have

$$
\begin{aligned}
C_{\varphi}^{*} f(w)=f(0) & +w \sum_{i=1}^{2}\left(f\left(\sigma_{i}(w)\right) \frac{\sigma_{i}^{\prime}(w)}{\sigma_{i}(w)\left(1-\bar{a} \sigma_{i}(w)\right)}\right. \\
& \left.-f(0) \frac{\sigma_{i}^{\prime}(w)}{\sigma_{i}(w)}+\frac{\sigma_{i}^{\prime}(w)}{\sigma_{i}(w)-a}\left(f\left(\sigma_{i}(w)\right)-f(a)\right)\right),
\end{aligned}
$$

and for $w=0, C_{\varphi}^{*} f(0)=f(0)$. The statement for arbitrary $f$ in $\mathcal{D}$ follows from the density of the polynomials.

Theorem 4.4. Let $\varphi$ be a finite Blaschke product, say of degree $n$. Then the formula for the adjoint of $C_{\varphi}$ on the Bergman space is given by

$$
C_{\varphi}^{*} f(w)=\sum_{k} \operatorname{Res}\left(\frac{F(z) \varphi^{2}(z)}{z^{2}(\varphi(z)-w)^{2}}, z_{k}\right)
$$

where $F(z)$ is holomorphic on the unit disk $\mathbb{U}$, such that for each $z \in \mathbb{U}, F^{\prime}(z)=f(z)$, and the sum is taken over the zeros $z_{k}$ of the denominator of the $\varphi^{2}(z)$ and the zeros of the rational function $z^{2}(\varphi(z)-w)^{2}$.

Proof. It suffices again to prove the statement only when $f$ is a polynomial. Each finite Blaschke product $\varphi$ is analytic on a larger disk and $|\varphi(z)|=1$ on $T$. So, 
from the Residue theorem, we get

$$
\begin{aligned}
C_{\varphi}^{*} f(w) & =\int_{D} \frac{f(z)}{(1-\overline{\varphi(z)} w)^{2}} d A(z) \\
& =\int_{D} \frac{F^{\prime}(z)}{(1-\overline{\varphi(z)} w)^{2}} d A(z) \\
& =\int_{T} \frac{F(z)}{(1-\overline{\varphi(z)} w)^{2}} \frac{d z}{2 \pi i z^{2}} \\
& =\int_{T} \frac{F(z) \varphi^{2}(z)}{z^{2}(\varphi(z)-w)^{2}} \frac{d z}{2 \pi i^{\prime}}
\end{aligned}
$$

and the statement follows from the Residue theorem since the only poles in $\mathbb{U}$ of the integrand are the zeros of the denominator and the zeroes of the denominator of $\varphi^{2}(z)$. The statement for arbitrary $f$ in $\mathcal{A}^{2}$ follows from the density of the polynomials.

Example 4.5. Let $\varphi(z)=\prod_{j=1}^{2} \frac{z-a_{j}}{1-\overline{a_{j}} z}$, with $a_{j} \in \mathbb{U}$, and $f \in \mathcal{A}^{2}$. Then we have

$$
C_{\varphi}^{*} f(w)=\int_{T} \frac{F(z) \varphi^{2}(z)}{z^{2}(\varphi(z)-w)^{2}} \frac{d z}{2 \pi i},
$$

where $F(z)$ is holomorphic on the unit disk $\mathbb{U}$, such that for each $z \in \mathbb{U}, F^{\prime}(z)=f(z)$.

Now suppose $w_{0} \in \mathbb{U}$ is a regular value of $\varphi$ and $V \subset \mathbb{U}$ is any connected neighborhood of $w_{0}$ on which are defined 2 distinct branches $\left\{\sigma_{j}\right\}_{j=1}^{2}$ of $\varphi^{-1}$. Then by a similar computation as example (4.3), for all nonzero $w \in V$,

$$
\begin{aligned}
C_{\varphi}^{*} f(w)=w^{2}\left(\sum_{i=1}^{2}\left(\frac{\sigma_{j}^{\prime 2}(w)}{\sigma_{j}^{2}(w)} f\left(\sigma_{j}(w)\right)\right)+\right. & \\
& \left.2 \frac{\sigma_{1}^{\prime}(w) \sigma_{2}^{\prime}(w)}{\sigma_{1}(w) \sigma_{2}(w)} * \frac{\sigma_{2}(w) F\left(\sigma_{2}(w)\right)-\sigma_{1}(w) F\left(\sigma_{1}(w)\right)}{\sigma_{2}(w)-\sigma_{1}(w)}\right),
\end{aligned}
$$

and for $w=0, C_{\varphi}^{*} f(0)=f(0)$.

Theorem 4.6. Let $R$ be any rational univalent holomorphic self-map of the closed disk. Define the associated rational function $R^{*}$ as Theorem 4.1. Then the following formula for the adjoint of $C_{R}$ on the Bergman space $\mathcal{A}^{2}$ holds:

$$
C_{R}^{*} f(w)=\sum_{k} \operatorname{Res}\left(\frac{F(z)}{z^{2}\left(1-R^{*}(z) w\right)^{2}}, z_{k}\right),
$$

where the sum is taken over those zeros $z_{k}$ of rational function $z^{2}\left(1-R^{*}(z) w\right)^{2}$ which belong to $\mathbb{U}$, and $F(z)$ is holomorphic on the unit disk $\mathbb{U}$, such that for each $z \in \mathbb{U}$, $F^{\prime}(z)=f(z)$. 
Proof. Let $f$ first be a polynomial, and $\mathrm{F}$ be holomorphic on $\mathbb{U}$ such that for each $z \in \mathbb{U}, F^{\prime}(z)=f(z)$. Since every univalent holomorphic self-map of $\mathbb{U}$ induces a bounded composition operator on $\mathcal{A}^{2}$, we have

$$
\begin{aligned}
C_{R}^{*} f(w) & =\int_{D} \frac{f(z)}{(1-\overline{R(z)} w)^{2}} d A(z) \\
& =\int_{D} \frac{F^{\prime}(z)}{(1-\overline{R(z)} w)^{2}} d A(z) \\
& =\int_{T} \frac{F(z)}{\left(1-\overline{R\left(\frac{1}{\bar{z}}\right)} w\right)^{2}} \frac{d z}{2 \pi i z^{2}} \\
& =\int_{T} \frac{F(z)}{z^{2}\left(1-R^{*}(z) w\right)^{2}} \frac{d z}{2 \pi i} \\
& =\sum \operatorname{Res}\left(\frac{F(z)}{z^{2}\left(1-R^{*}(z) w\right)^{2}}, z_{k}\right),
\end{aligned}
$$

where the sum is taken over the zeros $z_{k}$ of the rational function $z^{2}\left(1-R^{*}(z) w\right)^{2}$. The statement for arbitrary $f$ in $\mathcal{A}^{2}$ follows from the density of the polynomials.

\section{Acknowledgement}

We are grateful to the referee for his or her valuable comments.

\section{References}

[1] A. Abdollahi, Self-commutators of composition operators on the Dirichlet space, Proc. Amer. Math. Soc. Vol. 136 (2008), 3185-3193.

[2] P. S. Bourdon, D. Levi, S. K. Narayan, and J. H. Shapiro, Which linearfractional composition operators are essentially normal?, J. Math. Anal. App. 280 (2003) 30-53.

[3] P. S. Bourdon and B. D. MacCluer, Selfcommutators of automorphic composition operators, Complex Var. Elliptic Equ. 52 (2007), 85-104.

[4] P. S. Bourdon, J. H. Shapiro, Adjoints of rationally induced composition operators, J. Functional Analysis (2008), 1995-2012.

[5] G. A. Chacón and G. R. Chacón, Some properties of composition operators on the Dirichlet space, Acta Math. Univ. Comenian. (N.S.) 74 (2005), no. 2, 259-272.

[6] C. Cowen, Linear fractional composition operators on $H^{2}$, Integral Equations Operator Theory 11 (1988) 151-160.

[7] C. Cowen and B. D. MacCluer, Composition Operators on Spaces of Analytic Functions, CRC Press, Boca Raton, FL, 1995. 
[8] E. A. Gallardo-Gutirrez and A. Montes-Rodríguez, Adjoints of linear fractional composition operators on the Dirichlet space, Math. Ann. 327 (2003), no. 1, 117134.

[9] C. Hammond, The norm of a composition operator with linear symbol acting on the Dirichlet space, J. Math. Anal. Appl. 303 (2005), no. 2, 499-508.

[10] M. J. Martin and D. Vukotic, Norms and spectral radiu of composition operators acting on the Dirichlet spaces, J. Math. Analysis and Application 304 (2005), 22-32.

[11] M. J. Martin and D. Vukotic, Adjoints of composition operators on Hilbert spaces of analytic functions, J. Funct. Anal. 238 (2006), no. 1, 298-312.

[12] J. H. Shapiro, Composition Operators and Classical Function Theory, Springer-Verlag, 1993.

[13] R. K. Singh and J. S. Manhas, Composition Operators on Function Spaces, North-Holland Publishing Co., Amsterdam, 1993.

Department of Mathematics, Shiraz University,

Shiraz, IRAN

emails:abdollahi@shirazu.ac.ir, tahereh136355@yahoo.com

Department of Engineering, Khonj Branch,

Islamic Azad University,

Khonj, IRAN

email:math.samira@yahoo.com 